A R T I G O

$\infty$

\title{
O Futuro da Publicação Científica
}

\section{Adeilton Brandão ${ }^{a}$}

Pesquisador Titular do Laboratório Interdisciplinar de Pesquisas Médicas e Editor das Memórias do Instituto Oswaldo Cruz, Instituto Oswaldo Cruz - Fiocruz. Av. Brasil, 4.365. Rio de Janeiro, RJ, E-mail: adeilton.brandao@fiocruz.br.

\section{Resumo}

Atualmente, existem mais de 32.000 revistas cientificas, que, juntas, publicam por ano mais de 1.000 .000 de artigos em todas as áreas do conhecimento. Isso implica que uma grande quantidade de editores e revisores, além de mais tempo e recursos, serão progressivamente necessários para avaliar adequadamente os resultados científicos apresentados nesses artigos. Até o presente, esse trabalho seletivo de editores e revisores não tem sido suficiente para separar a ciência produzida sob as mais rigorosas práticas daquela feita sem rigor e sem qualidade. Alia-se a isto um outro problema: quem deve pagar a conta da divulgaçáo dos resultados da pesquisa ? Náo há, por enquanto, uma resposta direta a esta questão, haja vista as várias opçôes à disposição de cientistas e seus financia- dores. A explicitação dessas questôes nos leva a uma outra reflexão mais ampla e de muita incerteza: o que esperar para o futuro da publicação cientifica? Assumindo como ponto de partida algumas tendências do atual ambiente editorial científico, é possível imaginar um cenário para as próximas décadas que inclua inovaçóes nos seguintes temas: a) auto publicação; b) repositórios de "preprint"; c) compartilhamento de dados.; d) revisão por pares aberta, massiva e descentralizada; e) plataforma institucional de publicação. Adicionalmente, pode-se imaginar um outro cenário muito rompedor de padróes no qual uma atividade estritamente conduzida por seres humanos seria substituída por algoritmos: a revisão massiva e descentralizada de artigos científicos. Não há nenhuma garantia de que esses cenários se consolidem, mas apenas uma certeza: o atual ambiente de publicação científica modificar-se-á bastante nos próximos anos em função de avanços tecnológicos e as mudanças sociais, políticas e econômicas que estão acontecendo neste momento. Editores, cientistas, financiadores e respectivas instituiçôes devem desde já 
incluir estas tendências nas suas agendas de discussão e planejamento.

\section{Palavras-chave}

Publicação científica; revisão por pares; cenários futuros; integridade científica; prática editorial; preprint.

\section{Abstract}

Currently, there are more than 32,000 scientific journals that, together, publish more than 1,000,000 articles per year in all areas of knowledge. This implies that a greater number of editors and reviewers, in addition to more time and resources, will progressively be needed to properly evaluate the scientific results presented in these articles. To date, this selective work by editors and reviewers has not been sufficient to separate the science produced under the most rigorous practices from that done without rigor and without quality. This is coupled with another problem: who should pay the bill for the dissemination of research results? There is as yet no direct answer to this question, given the various options available to scientists and their funders. The explanation of these questions leads us to another broader and very uncertain reflection: what to expect for the future of scientific publication? Taking as a starting point some trends in the current scientific editorial environment, it is possible to imagine a scenario for the coming decades that includes innovations in the following themes: a) self-publication;

b) "preprint" repositories; c) data sharing; d) open, massive and decentralized peer review; e) institutional publishing platform. Additionally, one can imagine another very pattern-breaking scenario in which an activity strictly conducted by human beings would be replaced by algorithms: the massive and decentralized review of scientific articles. There is no guarantee that these scenarios will consolidate, but just a certainty: the current scientific publication environment will change significantly in the coming years due to technological advances and the social, political and economic changes that are taking place at this time. Editors, scientists, financiers and their respective institutions should now include these trends in their discussion and planning agendas.

\section{Keywords}

Scientific publication; peer review; future scenarios; scientific integrity; editorial practice; preprint.

\section{O CENÁRIO ATUAL DA PUblicaÇÃo CIENTÍFICA}

Considerando todas as áreas do conhecimento, o número de revistas cientificas existentes hoje é superior a 32.000 (de acordo com as informaçóes das bases de revistas 
"Scopus" - https://www.scopus.com/ - e "Directory of Open Access Journals" https://doaj.org/). Este conjunto de revistas publica anualmente bem mais de 1.000 .000 de artigos e, essencialmente, atende a uma demanda de publicação crescente por parte dos cientistas. Além do aspecto positivo que isso representa (um grande numero de revistas obviamente contribuem para uma maior disseminação do conhecimento gerado), há um efeito menos interessante dessa grande oferta de revistas e respectiva demanda de publicação de artigos: um ônus sobre aqueles procedimentos editoriais que buscam conferir qualidade e credibilidade aos dados científicos a serem publicados. Para lidar com esse imenso volume de artigos são necessários mais editores, mais revisores (pessoas importantíssimas neste processo), mais tempo e recursos para avaliar adequadamente as evidências cientificas. $O$ que se percebe até agora é que o processo não está sendo eficaz para separar a ciência conduzida sob as mais rigorosas práticas daquela feita sem rigor, sem qualidade e sem respeitar a integridade cientifica $^{1,2,3}$. Adicionalmente, há os interesses econômicos que também podem perturbar essa delicada relação entre a boa divulgação do conhecimento cientifico (que obviamente tem um custo financeiro!) e a simples veiculação de artigos feita para "gerar dinheiro" (revistas que não se alinham às boas práticas da publicação cientifica e nem observam os princípios da integridade cientifica $)^{4}$.
Além dos meios materiais e financeiros $^{5}$, uma revista científica não subsiste por muito tempo se os editores, revisores e autores não contam com o reconhecimento social e fraternal e respeito pelo exercício de sua atividade. Sem este reconhecimento, náo ocorre o estimulo para a consolidação de um ciclo virtuoso: trabalho e dedicação que proporcionam reconhecimento, que por sua vez estimula mais trabalho e dedicação, que trazem mais reconhecimento. Este ciclo é um dos eixos motivadores das pessoas em atividade de pesquisa cientifica!

Os eixos motivadores da pesquisa cientifica devem se materializar quando se estabelecem os "processos institucionais", isto é, açôes decorrentes menos do empenho de poucos indivíduos que do planejamento e execução de estratégias nacionais coordenadas por instituições que são perenes à sucessão dos governos. Por exemplo, a criação do CNPq e da CAPES no início da década de 1950 (e mais adiante, das agencias estaduais de fomento à pesquisa) impulsionou as açóes individuais (ou de centro de pesquisas) que buscavam isoladamente o desenvolvimento cientifico do Brasil. Esta coordenação também contribuiu muitíssimo para o aprimoramento da prática editorial cientifica brasileira, culminando entre outras iniciativas, na criação da biblioteca eletrônica de revistas cientificas, atualmente denominada de Scielo (https://scielo.org/pt). Foi um importante marco para a publicação cientifica no 
Brasil e a América Latina, pois a Scielo também agrega revistas de outros países da região, proporcionando uma estrutura de apoio, melhoramento e monitoramento contínuo das revistas cientificas albergadas.

Um dos principais problemas a serem resolvidos na divulgação científica é o financiamento do custo de publicação dos artigos. Este problema pode ser resumido na seguinte pergunta: quem deve pagar a conta para a divulgação dos resultados da pesquisa de qualidade?

No momento não há clareza sobre essa questão, haja vista as diversas opçóes que disputam a atenção de cientistas e seus financiadores, a saber:

i) o leitor paga para acessar o artigo. Este é o modelo "tradicional por assinatura"; na prática são as bibliotecas das universidades que pagam pelo acesso, sendo que nos últimos anos este pagamento pesa bastante no orçamento destas instituições;

ii) o autor paga para publicar (modelo recente, tem a vantagem de garantir o acesso aberto mas o custo pode ser muito elevado para os autores individualmente);

iii) a instituição de pesquisa (universidades, centros de pesquisa) paga diretamente às revistas o custo de publicação dos artigos de seus pesquisadores. É interessante por ser acesso aberto, mas o custo ao longo do tempo pode se tornar elevado para estas instituiçóes que terão que separar mais uma fatia dos seus já comprometidos orçamentos.

iv) financiador dos projetos de pesquisa inclui o custo da publicação como item financiável no orçamento a ser aprovado para a execução do projeto. Todavia, o custo final pode ficar elevado se a previsão for de muitos artigos por projeto.

v) o financiador contrata um serviço de publicação científica de terceiros, criando uma "plataforma de publicação". Neste modelo, a agência de financiamento negocia o valor do gerenciamento de um grande volume de artigos a ser disponibilizado em uma plataforma de publicação própria. Com esta abordagem, a agencia reduz o custo de publicação, permite a divulgação imediata e garante ao público em geral o acesso livre aos resultados da pesquisa (modo acesso aberto, que pode evoluir para o modo "dados abertos").

O modelo de publicação (v) já é adotado por dois grandes financiadores de pesquisas científicas: a fundação Bill \& Melinda Gates (https://gatesopenresearch.org/), e a Wellcome Trust (https://wellcomeopen research.org/). Ambas possuem plataformas de publicação com as seguintes características:

i) os autores financiados por elas são obrigados a depositar os seus artigos nas plataformas (mandato de publicar em acesso aberto); 
ii) o artigo é imediatamente disponibilizado e acessível a todo leitor;

iii) a revisão pelos pares é aberta, e o parecer dos revisores fica disponível aos leitores;

iv) o artigo permanece na plataforma independente do resultado da avaliação pelos pares (no entanto, novas versóes com as alteraçóes sugeridas pelos revisores podem ser adicionadas);

v) o custo da publicação é coberto pela própria agência financiadora.

Apesar das nítidas vantagens do modelo de "plataforma de publicação", ele ainda é uma exceção no atual cenário editorial: no máximo, os organismos de financiamento da pesquisa científica recomendam o 'acesso aberto’ e aderência as boas práticas de publicação/integridade cientifica. No Brasil, por enquanto, não há nenhum movimento para implementação de tais plataformas de publicação ou discussóes públicas sobre como lidar com o custo (cada vez maior) da publicação de artigos científicos ${ }^{6}$.

Não obstante os benefícios do modelo de publicação em “acesso aberto", o mesmo trouxe consigo um efeito colateral relevante: ao exigir dos autores a "taxa de processamento de artigo" (i.e., pagar pela publicação), abriu-se uma grande oportunidade de negócios na editoração científica, uma atividade que antes era quase uma exclusividade de organizaçóes acadêmicas e de umas poucas empresas editoriais. Dada a virtual ausência de barreiras técnicas, regulatórias ou financeiras, criar uma revista para publicação de artigos transformou-se em uma atividade ao alcance de qualquer individuo com um investimento mínimo. Isso resultou no surgimento de conflitos de interesse náo explicitados (menor rigor na análise dos artigos resulta em número maior de unidades publicadas e mais "dinheiro no caixa") e na banalização da publicação científica ${ }^{4}$. Com tamanha oferta de revistas científicas, e sem nenhum controle, o resultado é um excesso de artigos científicos de qualidade duvidosa, que em termos da teoria da oferta e demanda, está apenas respondendo à pressão que os próprios pesquisadores sofrem no exercício da sua atividade profissional: o seu progresso na carreira e aprovação de novos financiamentos dependem em larga medida do número de artigos que conseguem publicar em um determinado período. Isto por sua vez alimenta a expansão de "novas" revistas científicas, sobrecarregando a revisáo pelos pares e aumentando o custo geral da publicaçáo e do próprio ato de "fazer ciência". É importante mencionar que a revisão pelos pares é uma atividade voluntária, sem remuneração, cujo valor é capturado integralmente pelos "controladores das revistas". Isto ocorre porque os revisores são também pesquisadores que deixam de fazer as suas atividades remuneradas para "avaliar os artigos a pedido de um editor de uma revista” por um certo período de tempo. Há, portanto, um custo 
“implícito" na revisão pelos pares que não é pago pelos "controladores/proprietários" das revistas. Este custo acaba ficando a cargo das instituiçóes de pesquisa (que remuneram os pesquisadores) ou mesmo das agencias de financiamento. É preciso explicitar este custo, torná-lo transparente e adequadamente dividi-lo entre todos os envolvidos!

Portanto, um dos desafios para os que atuam no melhor interesse da divulgação científica é "como alinhar" esta quantidade de revistas e artigos às necessidades de qualidade, rigor e relevância da prática científica.

\section{TendênCias para o Futuro}

A retrospectiva das previsões (ou projeçôes) elaboradas para o futuro em qualquer ramo da atividade humana (e em qualquer época) aponta duas certezas:

vi) as experiências individuais e coletivas no cotidiano daquele cenário futuro previsto (projetado) serão diferentes daquelas vividas no momento em que o exercício de futuro foi realizado.

vii) as previsões vão falhar!

Há uma variedade de relatos descrevendo como as previsóes elaboradas por destacados líderes empresariais, economistas, cientistas, analistas políticos etc se mostraram completamente equivocadas ${ }^{7,8,9}$. Se é assim, qual é a motivação para se dedicar tempo a uma atividade que está fadada ao fracasso desde o seu começo? Talvez a melhor resposta a esta pergunta deva ser dada pelos filósofos, pois trata-se de uma atividade que os seres humanos nunca deixaram de executar, apesar da taxa de sucesso ser praticamente nula. Genericamente, podemos dizer que se trata da "esperança" de que os fatos possam ser melhor controlados se pudermos de alguma forma antecipar as suas consequências. Mas, historicamente, as evidências tem nos mostrado que ainda náo subimos de nível neste "patamar de esperanças" das previsões!

Há, porém, uma outra motivação de natureza mais pragmática: o ato de elaborar previsôes ou projeçôes para o futuro sobre qualquer atividade humana é por si só um interessante desafio intelectual. A construção de um cenário possível (ou desejável) para um tempo muito adiante do atual, exige conhecer os antecedentes da atividade em análise, as suas condiçóes no presente, e o conjunto de fatores que influenciam direta e indiretamente os resultados práticos desta atividade. Tudo isso, somado ao esforço de se projetar algo no desconhecido (o futuro) resulta em aquisição de conhecimento e acumulo de informação. Certamente, os autores desse exercício intelectual e aqueles que vão receber ou analisar o seu resultado terão sido expostos a algo novo, e como sabemos, as coisas novas podem mobilizar (e inspirar) as pessoas para outras ações e direçôes. 
Assumindo que o propósito da tentativa de "antecipar o futuro" é listar as alternativas de "desdobramentos possíveis e desejáveis" para eventos atuais, o que esperar então do "ambiente de publicação científica" para as próximas décadas? $\mathrm{O}$ máximo que podemos fazer aqui é um sumário das "nossas esperanças" tendo como ponto de partida as tendências que já observamos no meio editorial científico. Nesse caso, considera-se como "tendências" as iniciativas que rompem com certos padróes do ambiente de publicação cientifica. Ressalte-se que não, há neste momento, nenhuma garantia de que essas iniciativas consolidar-se-ão nos médio e longo prazos, pois muitas delas estão aproveitando as imensas transformaçóes e oportunidades que as tecnologias digitais nos propiciam. Dentre estas tendências, vale mencionar:

i) a auto publicação. Cientistas definem "quando, como, onde" publicar o resultado das suas pesquisas, em seus próprios termos e demandas de tempo, funcionando também como "editores" dos seus próprios artigos ao convidando os seus pares para ler, criticar, analisar, replicar e reutilizar os dados obtidos. Esta iniciativa pressupóe que uma parte dos problemas vistos atualmente na publicação científica decorre de um fator: a corrida acelerada para as poucas revistas de prestigio que resultam no funil da publicação de artigos. Exemplo: Researchers.one, the winnower; ii) repositório "preprint". Os artigos são imediatamente disponibilizados aos leitores, sem revisão pelos pares. Idealmente, a própria comunidade de cientistas deveria realizar espontaneamente a leitura critica de cada artigo submetido ao repositório. Os comentários, criticas e sugestóes de alterações/correções seriam acolhidos pelos autores e integrados a novas versóes do artigo. Esse processo repetir-se-ia enquanto houvesse necessidade de alteraçóes no texto. Ex.: Biorxiv, Medrxiv, Scielo Preprints;

iii) compartilhamento de dados: os dados primários obtidos pelos pesquisadores sãoimediatamentedisponibilizadosatravés de plataformas com acesso livre. Os dados científicos ali depositados podem ser analisados, reutilizados, comentados, citados, incorporados em outro conjunto de dados para teste de hipóteses etc. Tudo isso com reconhecimento e devido crédito aos pesquisadores que geraram originalmente os dados. Ex.: os bancos de dados de genomas, transcriptomas e proteomas já funcionam dessa forma. Busca-se ampliar este conceito para outros tipos de dados de pesquisa. A iniciativa Fairsharing oferece várias recomendaçóes para o compartilhamento e o uso de dados (https://fairsharing. org/);

iv) revisão pelos pares aberta, massiva e descentralizada: ao contrário do prati- 
cado atualmente pela maioria das revistas científicas, avaliação crítica feita por centenas de pesquisadores aos artigos de outros colegas será totalmente aberta, com a divulgação integral das conversas, discussóes e comentários envolvendo o conteúdo do artigo (ou somente dos dados, se a tecnologia for utilizada para avaliação qualitativa dos dados originais de pesquisa). Algumas revistas já praticam este tipo de revisão aberta, mas ainda é um número bem reduzido e limitado apenas ao parecer de dois ou três revisores, sem o aporte de tecnologias de análise em massa;

v) plataforma institucional de publicação: as instituiçóes que financiam a pesquisa ou os centros que desenvolvem projetos de pesquisa poderão criar os seus próprios veículos de divulgação científica. Nestes veículos se juntam numa mesma estrutura tecnológica o conceito de "revista" e "repositório": autores submetem seus artigos com acesso imediato e revisão aberta pelos pares ('revista'). Esses artigos permanecerão para sempre nessa plataforma, sofrendo apenas o acréscimo de novas versóes. Ao receberem parecer positivo dos revisores, os artigos são indexados (identificados como "publicados") pelos buscadores de artigos.

\section{A REVISÃo MASSIVA, DESCENTRALIZADA \\ E AUTOMATIZADA DE ARTIGOS EM REPOSITÓRIOS: UM CENÁRIO POSSÍVEL?}

Considerando a evolução tecnológica e o desenvolvimento de sofisticados algoritmos para tratamento de quantidades massivas de dados, podemos imaginar um cenário no qual a revisão pelos pares, em qualquer das suas variaçóes, venha a ser substituído por robôs de revisão. Assim, poderá ser desenvolvida uma plataforma autônoma na qual os autores submetem os seus artigos, e os algoritmos processam este manuscrito utilizando critérios que incluem índices de qualidade (nova informação, novo protocolo, novo método etc), parâmetros quantitativos (impacto esperado - por exemplo, algoritmos que permitem prever o número de citaçôes provável para um determinado autor), e produzem uma estimativa da novidade dos dados descritos e seu potencial para reprodução/replicação.

Considerando ainda que a análise de big data já é uma tarefa rotineira (por exemplo, algoritmos do Google e outras organizaçóes diariamente inspecionam, rastreiam e coletam informaçóes de todos os computadores conectados em rede mundial), chegará o momento em que um robô processará toda a informação disponível em todas as áreas do conhecimento e fará uma comparação dessa massa de informaçóes com o conteúdo de qualquer manuscrito subme- 
tido a um repositório, permitindo dizer se este trabalho tem ou não algo de original em termos estritamente científicos. Quando isto acontecer, é possível assumir que a ação humana na revisão pelos pares será reduzida. Resta-nos agora perguntar se este processo de revisão pelos pares, que hoje é totalmente humano, poderá ser integralmente substituído por inteligência artificial.

Neste momento, caberá de novo questionarmos os filósofos:

"devemos delegar para as máquinas uma atividade tipicamente humana como a prática cientifica?"

\section{REFERÊNCIAS}

1. Glasziou P, Altman DG, Bossuyt P, et al. Reducing waste from incomplete or unusable reports of biomedical research. Lancet. 2014;383(9913):267-276. doi: 10.1016/S0140-6736(13)62228-X

2. Smaldino PE, McElreath R: The natural selection of bad science. R Soc Open Sci. 2016;3(9):160384. doi: 10.1098/ rsos. 160384

3. Rennie D: Integrity in Scientific Publishing. Health Serv Res. 2010 45(3): 885-896. doi: 10.1111/j.14756773.2010.01088.x

4. Grudniewicz A. et al. 2019. Predatory journals: no definition, no defence.
Nature, 576: 2019. https://www.nature. com/articles/d41586-019-03759-y

5. Odell J, Whipple EC: The changing landscape of scholarly publishing: will Radiation Research survive?. Radiat Res. 2013; 180 (4): 335-339. doi: https:// doi.org/10.1667/RR3528.2

6. Brandao A: Midpoint: the publishing platform as a hybrid between a preprint server and a peer reviewed journal. Mem. Inst. Oswaldo Cruz - Blog. 2019. https://memorias.ioc.fiocruz.br/ recent-posts/item/43-midpoint-thepublishing-platform-as-a-hybridbetween-a-preprint-server-and-a-peerreviewed-journal).

7. Epstein D: The peculiar blindness of experts. The Atlantic. 2019. https:// www. theatlantic.com/magazine/ archive/2019/06/how-to-predictthe-future/588040/

8. Makridakis S, Hogarth RM, Gaba A: Why Forecasts Fail. What to Do Instead. MIT Sloan Management Review. 2010. https://sloanreview.mit.edu/article/ why-forecasts-fail-what-to-do-instead/

9. Lavenda D: My Prediction for Technology in 2020? Predictions Will Fail. CMSWire. 2019. https://www. cmswire.com/digital-workplace/myprediction-for-technology-in-2020predictions-will-fail/ 